\title{
Comodidad de los pacientes hospitalizados con cancer en dos instituciones hospitalarias, Cartagena
}

\section{Comfort of patients suffering from cancer hospitalized in two health facilities, Cartagena}

\author{
Amparo Montalvo', Boris Cabrera², José Contreras², Sandra Quiñonez ${ }^{3}$, Eileen Salem³, \\ Carolay Serpa ${ }^{3}$
}

\section{Resumen}

Objetivo: Determinar el nivel de comodidad de los pacientes hospitalizados con cáncer en dos instituciones de salud de Cartagena. Metodología: Estudio descriptivo, realizado $\mathrm{Al}$ total de pacientes hospitalizados con cáncer en dos instituciones de salud. Para recolectar la información se utilizó una encuesta socio-demográfica y el cuestionario que mide comodidad física, psicoespiritual, social y ambiental elaborado por Kolcaba. Resultado: La edad promedio de los participantes fue de 50 años (D.E. $=18,7$ ), hombres en un $52.8 \%$. En la dimensión física, social y ambiental el confort predominante fue la trascendencia en el $99.4 \%$, $100 \%$ y $51.1 \%$ respectivamente. Con respecto a la dimensión psicoespiritual predominó la tranquilidad como confort en un 61,7\%. Conclusiones: Los participantes del estudio fueron hombres, bachilleres, católicos, citadinos, viven en pareja y pertenecen a un estrato socioeconómico bajo. La religión es una estrategia de apoyo para ellos. El estudio reportó la trascendencia, como comodidad general, que se constituye en el mejor nivel de confort en las dimensiones física y social, la tranquilidad se obtuvo en las dimensiones psicoespiritual y ambiental.

Palabras clave: Cáncer, Hospitalización, Bienestar. Calidad de vida Fuente: (DeCs, Bireme).

\section{Abstract}

Objective: To determine the extent of comfort of patients suffering from cancer hospitalized in two health facilities in Cartagena. Methodology: It was a descriptive study conducted on all patients suffering from cancer hospitalized in two health facilities. For gathering information it was administered a sociodemographic survey and a questionnaire measuring physical pyschospiritual, social and environmental comfort drawn up by Kolcaba. Result: Patients' average age was of 50 (D.E. $=18,7$ ), men by $52.8 \%$. In the physical, social and environmental dimension, the predominant comfort was transcendence by $99.4 \%$, $100 \%$ and $51.1 \%$ respectively. Regarding the psychospiritual dimension tranquility was preferred as comfort by $61.7 \%$. Conclusions: Patients who took part in the study were men, bachelors, Catholics, city dwellers, living with a partner and belong to a lower socioeconomic stratum. Religion is a strategic support for them. The study showed transcendence as general comfort what constitutes the best level of comfort in the physical and social dimension. Tranquility was shown in the psychospiritual and environmental dimensions.

Keywords: Cancer, Hospitalization, Wellbeing. Life Quality.

\section{Introducción}

El Ser Humano es más que una entidad biológica, es espíritu, alma, conciencia, mente, emociones y sentimientos, que para expresarse requiere de un cuerpo, y se manifiesta en distintos planos, niveles y vibraciones $^{[1]}$. El individuo, es una totalidad imposible de separar en sus dimensiones, ya que no es fácil establecer cuánto influye una sobre las otras o cuánto depende la una de las otras frente al proceso salud-enfermedad ${ }^{[2]}$. En un proceso patológico como es la enfermedad crónica, ésta es concebida como un estado, que se caracteriza por la indisposición e incapacidad asociada a la presencia de una

1. Enfermera Docente Universidad de Cartagena, Departamento de Investigación y Educación Facultad de Enfermería, Universidad de Cartagena, Colombia

2. Estudiante Enfermería, Universidad de Cartagena, Colombia

3. Enfermera, Universidad de Cartagena, Colombia

Datos de contacto: Amparo Montalvo, correo electrónico: Amontalvop1@unicartagena.edu.co

Recibido: 07/06/2014 - Revisado: 08/06/2014 - Aceptado: 30/06/2014 
alteración patológica prolongada e irreversible, que implica incapacidad residual ${ }^{[3]}$. La cronicidad es continua y cambiante, tiene ritmos alternantes e imprime modificaciones en la vida diaria, en todas las dimensiones del ser humano ${ }^{[3]}$. La presencia de enfermedades como las cardiopatías, los accidentes cerebrovasculares y el cáncer, además de afectar las diferentes dimensiones del ser humano, también pueden afectar las etapas del ciclo vital humano; de ellas se destaca el cáncer como una de las enfermedades crónicas más prevalentes en el mundo ${ }^{[4]}$.

Según la OMS el cáncer es una de las primeras causas de muerte a nivel mundial; en 2008 se le atribuyeron 7,6 millones de muertes (aproximadamente, el 13\% del total). Las proyecciones de las cifras de incidencia de cáncer indican un incremento futuro de estas cifras en todo el mundo, este envejecimiento es heterogéneo, se está produciendo a un ritmo más rápido que el registrado históricamente en los países desarrollados, ocurre en un contexto caracterizado por una gran incidencia de la pobreza y una persistente y aguda inequidad social ${ }^{[5]}$. La Organización Panamericana de la salud estima que cada año en América Latina y el Caribe hay alrededor de 900.000 nuevos casos, 542.000 muertes, y más de 2 millones de personas que conviven con el cáncer ${ }^{[6]}$.

En un estudio realizado en Colombia ${ }^{[7]}$, se encontraron 17420 casos nuevos para los hombres y 21395 casos para las mujeres. Anualmente 10505 hombres y 10184 mujeres mueren a causa de diversos tipos de cáncer, siendo el de próstata y el de mama los canceres con más incidencia, estos resultados confirman los datos encontrados en el estudio realizado en el 2006 por parte del Instituto Nacional de cancerología en el departamento de Bolívar que encontró un aumento anual de 1057 casos nuevos para hombres y 1214 casos para mujeres, siendo la próstata y mama los de mayor aumento ${ }^{[8]}$. En Cartagena de Indias según el estudio de registro poblacional de cáncer hay una incidencia anual de 2065 casos distribuidos con un $62 \%$ en las mujeres y un $38 \%$ en hombres ${ }^{[9]}$.

La presencia de una enfermedad crónica discapacitante, genera en el paciente diferentes necesidades de salud, las cuales requieren ser satisfechas para el desarrollo de sus actividades habituales. Kolcaba $^{[10]}$ define las necesidades de cuidados de la salud como aquellas indispensables para conseguir la comodidad, a partir de las situaciones estresantes de cuidado de la salud, que los sistemas tradicionales de apoyo de los receptores no pueden satisfacer. Las medidas de confort las considera como intervenciones de enfermería diseñadas para satisfacer necesidades específicas de comodidad de las personas receptoras de cuidado (pacientes) ${ }^{[11]}$

Estas medidas son el resultado de las acciones proporcionadas por los profesionales de la salud, especialmente los pertenecientes a la disciplina de enfermería que gracias a la identificación de las necesidades y satisfacción de ellas, proporcionan alivio, tranquilidad y transcendencia ya sea en el contexto social, físico, ambiental y psicoespiritual e influyen en la comodidad ${ }^{[10]}$; de tal manera que se asocia con la actividad de cuidado que se le brinda al paciente ${ }^{[11]}$.

Al definir el concepto de comodidad, Kolcaba le encontró tres sentidos, desarrollados en cuatro contextos. El sentido de alivio se refiere a la satisfacción de necesidades específicas y se constituye en un estado que le permite al paciente transmitir y comunicar sus necesidades emocionales y espirituales cuando experimenta este sentimiento $^{[12]}$, el sentido de tranquilidad hace referencia al estado de calma de la persona quien demuestra aceptación de su condición, es una referencia a la comodidad de la mente y el cuerpo, es un estado positivo y el sentido de trascendencia se refiere a un estado en que se está por encima de los problemas o el dolor propio ${ }^{[12]}$.
Kolcaba ${ }^{[10]}$ en las fuentes teóricas del confort, conceptualiza la comodidad abordando las necesidades para tres tipos de confort (Alivio, Tranquilidad y Trascendencia) y la desarrolla en cuatro contextos diferentes (Físico, Psicoespiritual, Social y Ambiental), razón por la cual se entiende que la responsabilidad de brindar comodidad no es solo de la disciplina de enfermería, sino que es un trabajo en conjunto con las corporaciones, hospitales e instituciones prestadoras de servicios. El cumplimiento de estas condiciones debe generar inicialmente un mejor servicio, de atención para los receptores de cuidado, de mejores condiciones de aprendizaje para los estudiantes y de políticas claras de funcionamiento para la institución.

Flóre $z^{[13]}$ en su estudio expresa, que la hospitalización ocasiona un disconfort en todo el grupo familiar, lo que afecta su comodidad, la cual es un factor importante y relevante para el bienestar de un paciente y de su familia, situación de la que no están alejados los pacientes con cáncer. La hospitalización en estos pacientes ocasiona en algunos casos dificultades en la familia, por la necesidad de compañía de un familiar, lo que provoca estrés e interrumpe la función familiar y en otros la familia queda aislada y desinformada acerca de su pariente y no se le tiene en cuenta para el cuidado del mismo. Plantea además que las intervenciones de enfermería deben estar encaminadas a aumentar la comodidad del paciente, dando un giro total al enfoque del cuidado biológico y pasando a un enfoque centrado en el paciente y su familia.

Las intervenciones de enfermería están encaminadas a aumentar la comodidad del paciente y su familia ${ }^{[14]}$ y con el desarrollo del presente trabajo de investigación se busca identificar el nivel de comodidad de los pacientes hospitalizados con cáncer en dos instituciones hospitalarias, de manera que los resultados del estudio aporte información significativa para proporcionar comodidad con el cuidado de enfermería que requieren los pacientes hospitalizados con cáncer.

\section{Materiales y métodos}

Estudio descriptivo con abordaje cuantitativo, realizado al total de los pacientes hospitalizados por más de dos días en el servicio de oncología de dos instituciones hospitalarias de Cartagena. Participaron 180 pacientes adultos con diagnóstico de cáncer, clínicamente estables, con capacidad de responder las preguntas del cuestionario y desearan participar en el estudio. Se les informó acerca de la reserva de su identidad, de la protección de la información y el poder retirarse del estudio si lo consideraba necesario ${ }^{[15]}$. Se contó con el aval del Comité de Ética, la autorización de las instituciones y de los participantes al firmar el consentimiento informado.

La información fue recolectada por los investigadores, se aplicaron dos instrumentos, una encuesta socio-demográfica diseñada por los investigadores que consta de 14 ítems sobre nivel socioeconómico, escolaridad, estado civil, ocupación; características socioculturales (religión, escolaridad), geográficos (lugar de procedencia) y características relacionadas con la patología, y el cuestionario general sobre la comodidad propuesto por Kolcaba consta de 28 ítems $^{[12]}$. La confiabilidad del cuestionario general de comodidad está presentada en el coeficiente alfa de Cronbach es de 0,9, siendo utilizado en grupos de pacientes de oncología, médico-quirúrgicos, psiquiátricos y comunitarios $^{[13]}$.

El instrumento busca determinar el nivel de comodidad del paciente en cuatro dimensiones ${ }^{[12]}$, la física, que hace referencia a la condición fisiológica y homeostática del paciente e implica la rela- 
ción entre el "ser" y su nivel corporal; la social hace referencia a las relaciones de tipo interpersonal que sostiene el paciente, ya sea con su familia o en un círculo ampliado como son las sociales; la psicoespiritual se revisan aspectos que influyen en los estados emocionales del paciente y en la ambiental se evalúa el contexto en que se encuentra el paciente y la manera en que este se interrelaciona con la situación actual del individuo ${ }^{[12]}$.

La encuesta propuesta por Kolcaba ${ }^{[12]}$ es un instrumento de evaluación de 28 ítems, cada ítem se mide con una escala tipo Likert, con valores de 1 a 4, que corresponden a totalmente de acuerdo 4, de acuerdo 3, en desacuerdo 2 y totalmente en desacuerdo 1. En el presente estudio, para efectos de realizar el análisis de los resultados, los ítems contenidos en la escala fueron agrupados teniendo en cuenta los 3 tipos de confort estipulados por Kolcaba de la siguiente manera 1 para alivio, 2 y 3 para tranquilidad y 4 para trascendencia (Tabla 1).

Para el análisis estadístico del cuestionario general de comodidad se tuvo en cuenta que el puntaje mínimo total es de 28 puntos y el puntaje máximo de 112 . Se establecieron puntos de corte para cada una de la dimensiones en los tipo de alivio, tranquilidad y trascendencia. Para la dimensión física el punto de corte del tipo alivio fue de 0 a 6 , tranquilidad de 7 a 12 y trascendencia fue de más de 12; para la dimensión social el punto de corte del tipo alivio fue de 0 a 4 , tranquilidad 5 a 8 y trascendencia más de 8 ; para la dimensión psicoespiritual el punto de corte del tipo alivio fue 0 a 12 , tranquilidad 13 a 24 y trascendencia más de 24 y para la dimensión ambiental el punto de corte para el tipo alivio fue de Alivio 0 a 6 , tranquilidad 7 a 12, trascendencia más de 12.

Los datos fueron almacenados y analizados en el paquete estadístico STATA versión 10.0 de donde se obtuvieron frecuencias absolutas y relativas.

\section{Resultados}

Las características generales de los pacientes hospitalizados muestran como perfil que son hombres $(52,8 \%)$, con edad promedio de 50 años, que viven en pareja (60\%), católicos $(67,2 \%)$, bachilleres $(23,9 \%)$, de estrato socioeconómico bajo $(96,1 \%)$, procedentes del sector urbano $(57,2 \%)$, afiliados al régimen subsidiado $(69,44 \%)$.

\section{Dimensiones y tipos de comodidad}

Con base a los 3 tipos de confort (alivio, tranquilidad y trascendencia) estipulados por Kolcaba, circunscritos a las dimensiones física, social, psicoespiritual y ambiental; se encontró que el tipo de confort que predominó de manera general para las dimensiones física y social fue la trascendencia. En cuanto a la dimensión ambiental el tipo de confort predominante fue trascendencia y tranquilidad. Con respecto a la dimensión psicoespiritual el tipo de confort predominante fue tranquilidad y alivio (Tabla 2).

Dimensión física: Hace referencia a la condición fisiológica y homeostática del paciente e implica la relación entre el "ser" y su nivel corporal. Al preguntarle a los participantes acerca de si "El dolor que siento es difícil de soportar" se encontró que el 73,9\% (sumatoria de columnas 1 y 2) se muestra en desacuerdo con este enunciado; con relación al ítem "No me siento bien de salud" el $57,5 \%$ respondió estar en desacuerdo con lo expresado y en cuanto a la pregunta "Estoy muy cansado" el 82,8\% de la muestra manifestó estar en desacuerdo. A la pregunta "Estoy satisfecho" el 72,7\% (sumatoria de columnas 3 y 4 ) de las personas del estudio respondieron estar de acuerdo con este ítem (Tabla 3).

Tabla 1. Estructura taxonómica del confort de Kolcaba y Distribución de ítems por dimensiones del confort.

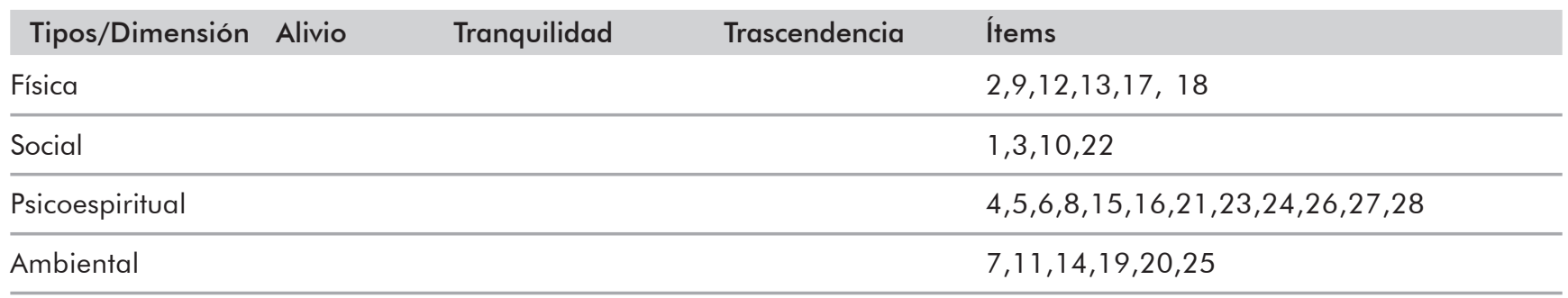

Fuente: Estructura taxonómica del confort de Kolcaba [16].

Tabla 2. Dimensiones y tipos de confort de Kolcaba en los pacientes hospitalizados con cáncer en dos instituciones hospitalarias, Cartagena.

\begin{tabular}{lcccccc} 
Tipos/Dimensiones & Alivio & $\%$ & Tranquilidad & Porcentaje & Trascendencia & Porcentaje \\
Física & 0 & 0,0 & 1 & 0,6 & 179 & 99,4 \\
\hline Social & 0 & 0,0 & 0 & 0,0 & 180 & 100,0 \\
\hline Psicoespiritual & 69 & 38,3 & 111 & 61,7 & 0 & 0,0 \\
\hline Ambiental & 0 & 0,0 & 88 & 48,9 & 92 & 51,1 \\
\hline General & 0 & 0,0 & 1 & 0,6 & 179 & 99,4 \\
\hline
\end{tabular}


Tabla 3. Tipo de confort alcanzado en la Dimensión física por los pacientes hospitalizados en dos servicios de Oncología en Cartagena.

\begin{tabular}{llcccccccc}
\multirow{2}{*}{ Pregunta } & Ítem & $\mathbf{7}$ & $\%$ & $\mathbf{2}$ & $\%$ & $\mathbf{3}$ & $\%$ & $\mathbf{4}$ & $\%$ \\
$\mathbf{2}$ & No quiero hacer ejercicio & 22 & 12,2 & 86 & 47,8 & 63 & 35,0 & 9 & 5,0 \\
\hline $\mathbf{9}$ & El dolor que siento es difícil de soportar & 34 & 18,9 & 99 & 55,0 & 42 & 23,3 & 5 & 2,8 \\
\hline 12 & Estoy duro del estómago & 22 & 12,2 & 88 & 48,9 & 61 & 33,9 & 9 & 5,0 \\
\hline 13 & No me siento bien de salud & 21 & 11,6 & 83 & 45,9 & 61 & 33,9 & 15 & 8,3 \\
\hline 17 & Estoy muy cansado & 39 & 21,7 & 110 & 61,1 & 29 & 16,1 & 2 & 1.1 \\
\hline $18 *$ & Estoy satisfecho & 9 & 5,0 & 40 & 22,2 & 96 & 53,3 & 35 & 19,4 \\
\hline
\end{tabular}

Fuente: Datos del estudio

Dimensión social: Al revisar los aspectos que conforman esta dimensión se encontró que el 56,6\% del grupo de estudio manifiesta estar en desacuerdo cuando se le pregunta si "Existen personas a las que puedo acudir si necesito ayuda"; con relación al enunciado "Mis amigos demuestran que se acuerdan de mí al enviarme tarjetas, visitarme o llamarme por teléfono" el 76,1\% de la población encuestada se muestra de acuerdo; con respecto a la pregunta "Me siento triste cuando estoy solo" el 76,7\% respondió estar en desacuerdo con lo expresado, y el 70,5\% de los participantes están en desacuerdo ante la afirmación "La situación en que estoy me preocupa" (Tabla 4).

Dimensión psicoespiritual: Ante la pregunta "Nadie me comprende" se encontró que el 67,2\% (sumatoria de columnas 1 y 2) se muestran en desacuerdo con este enunciado; con relación al ítem "Me aterra lo que va suceder" el 77,8\% respondieron estar en desacuerdo con lo mencionado; y en cuanto al enunciado, "He tenido muchos cambios" el 76,1\% de la población respondió estar en desacuerdo. El 70,6\% de la muestra manifestó estar en desacuerdo con el enunciado: "Me siento incapaz de controlarme".
Del total de personas encuestadas el 77,2\% (sumatoria de columnas 3 y 4), a la pregunta "siento confianza conmigo mismo" respondió estar de acuerdo; en cuanto al ítem "Me da aliento saber que me quieren" se encontró que el $86,7 \%$ de los participantes afirmaron estar de acuerdo; con respecto a "Me siento en paz conmigo mismo" y el $74,4 \%$ de la muestra expresó estar de acuerdo con este enunciado. Ante la pregunta "Estoy deprimido" solo el 56,1\% manifestó estar de acuerdo con el ítem y al revisar el punto "Le encontré significado a la vida" el $60 \%$ de los participantes expresaron estar de acuerdo (Tabla 5).

Dimensión ambiental: E1 71,1\% afirma estar en desacuerdo con la pregunta "El ruido no me deja descansar"; con relación al ítem "No me gusta este lugar" el $56,1 \%$ de la población encuestada manifestó estar en desacuerdo; ante la afirmación "Esta habitación me asusta" el 68,4\% responde estar en desacuerdo con lo mencionado, y al preguntarles sobre "Esta habitación huele muy mal", solo el $16,7 \%$ respondió estar en desacuerdo (Tabla 6).

Tabla 4. Tipo de confort alcanzado en la Dimensión social por los pacientes hospitalizados en dos servicios de Oncología en Cartagena.

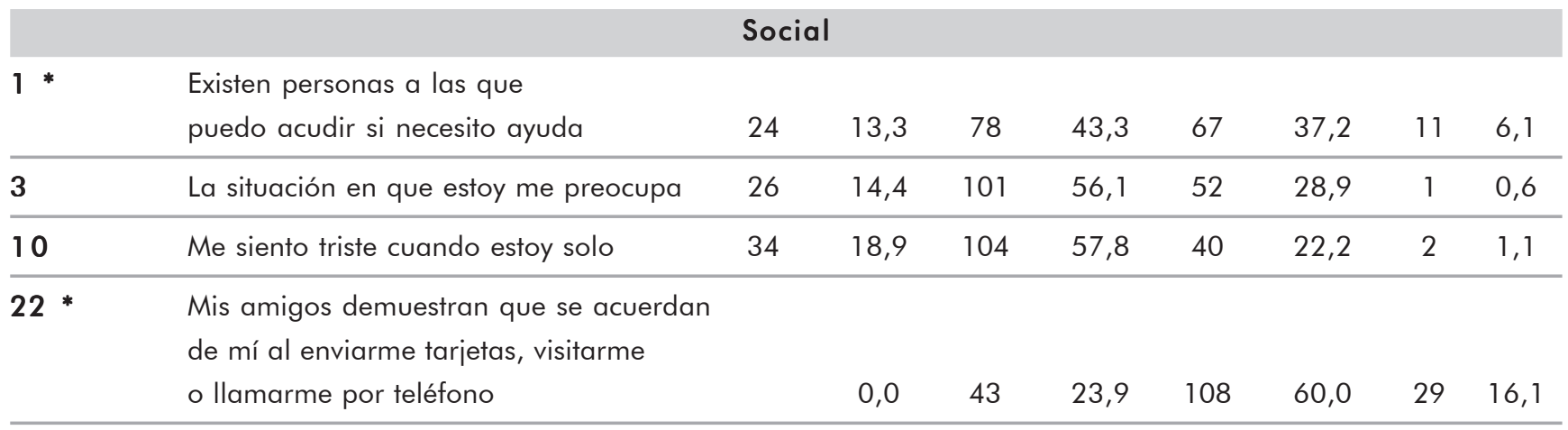

Fuente: Datos del estudio 
Tabla 5. Tipo de confort alcanzado en la Dimensión psícoespiritual por los pacientes hospitalizados en dos servicios de Oncología en Cartagena.

\begin{tabular}{|c|c|c|c|c|c|c|c|c|c|}
\hline \multicolumn{10}{|c|}{ Psicoespiritual } \\
\hline $4^{*}$ & Siento confianza conmigo mismo & 2 & 1,1 & 39 & 21,7 & 95 & 52,8 & 44 & 24,4 \\
\hline $5^{*}$ & Creo que mi situación actual es muy valiosa & 1 & 0,6 & 26 & 14,4 & 110 & 61,1 & 43 & 23,9 \\
\hline $6^{*}$ & Me da aliento saber que me quieren & & 0,0 & 24 & 13,3 & 111 & 61,7 & 45 & 25,0 \\
\hline 8 & Nadie me comprende & 26 & 14,4 & 95 & 52,8 & 45 & 25,0 & 14 & 7,8 \\
\hline 15 & Me aterra lo que va a suceder & 27 & 15,0 & 113 & 62,8 & 39 & 21,7 & 1 & 0,6 \\
\hline 16 & He tenido muchos cambios & 44 & 24,4 & 93 & 51,7 & 39 & 21,7 & 4 & 2,2 \\
\hline 21 & Aquí no me siento en el sitio correcto & 28 & 15,6 & 100 & 55,6 & 51 & 28,3 & 1 & 0,6 \\
\hline 23 & Necesito más y mejor información sobre mi salud & 35 & 19,4 & 87 & 48,3 & 47 & 26,1 & 11 & 6,1 \\
\hline 24 & Me siento incapaz de controlarme & 27 & 15,0 & 100 & 55,6 & 52 & 28,9 & 1 & 0,6 \\
\hline $26 *$ & Me siento en paz conmigo mismo & 3 & 1,7 & 43 & 23,9 & 96 & 53,3 & 38 & 21,1 \\
\hline 27 & Estoy deprimido & 11 & 6,1 & 68 & 37,8 & 76 & 42,2 & 25 & 13,9 \\
\hline $28 *$ & Le encontré significado a la vida & 9 & 5,0 & 63 & 35,0 & 85 & 47,2 & 23 & 12,8 \\
\hline
\end{tabular}

Fuente: Datos del estudio

Tabla 6. Tipo de confort alcanzado en la Dimensión ambiental por los pacientes hospitalizados en dos servicios de Oncología en Cartagena.

\begin{tabular}{|c|c|c|c|c|c|c|c|c|c|}
\hline \multicolumn{10}{|c|}{ Ambiental } \\
\hline 7 & El ruido no me deja descansar & 27 & 15,0 & 101 & 56,1 & 51 & 28,3 & 1 & 0,6 \\
\hline 11 & No me gusta este lugar & 12 & 6,7 & 67 & 37,2 & 76 & 42,2 & 25 & 13,9 \\
\hline 14 & Esta habitación me asusta & 30 & 16,7 & 93 & 51,7 & 50 & 27,8 & 7 & 3,9 \\
\hline 19 & Esta silla (cama) me lastima & 14 & 7,8 & 77 & 42,8 & 69 & 38,3 & 20 & 11,1 \\
\hline 20 & Mis artículos personales no están aquí & 23 & 12,8 & 86 & 47,8 & 62 & 34,4 & 9 & 5,0 \\
\hline 25 & Esta habitación huele muy mal & 37 & 20,6 & 113 & 62,8 & 30 & 16,7 & 0 & 0 \\
\hline
\end{tabular}

\section{Discusión}

En cuanto a las características sociodemográficas de los participantes del estudio se encontró que el promedio de edad fue de 50 años, dato similar al reportado por Payán [17] en un estudio exploratorio en el que participaron 50 pacientes con cáncer terminal y el promedio de edad fue de 52,6 años. Con respecto al sexo el 52,8\% de la muestra pertenece al género masculino, contrario a lo citado por Gaviria [18] quien reporta que el 60,7\% de los participantes fueron mujeres, en un estudio que evaluó la calidad de vida relacionada con la salud, el afrontamiento del estrés y las emociones negativas en pacientes con cáncer en tratamiento quimioterapéutico.

La religión que prevaleció fue la católica representada por el $67,2 \%$ de los participantes, seguida por la evangélica con el 14,4\% y los testigos de Jehová con el 13,9\%, Barbosa [19] reporta en su estudio de pacientes con cáncer de un hospital universitario en la ciudad de Bogotá predominó en un 76,3\% de la religión Católica, mientras que el 20,3\% corresponde a la religión Cristiana. La religión se utiliza como estrategia de afrontamiento en estos pacientes, ayudados por el conjunto de ritos y costumbres propias de cada una.

En cuanto a la dimensión física el tipo de confort que predomino en el 99,4\% de los participantes del presente estudio fue la trascendencia, información similar reporta Gómez [20] en un estudio donde participaron 77 pacientes con cáncer terminal, en el cual se encontró que los pacientes disfrutaban de un alto grado de bienestar físico con un puntaje de 21,22 en una escala de 0 a 28. Esta información indica que a pesar de las condiciones de salud en que se 
encuentran, los pacientes aceptan su condición de enfermedad y se ajustan a su estado.

Con relación a la dimensión social el tipo de confort que predominó fue la trascendencia en el 100\% de los participantes del estudio. Este dato es contrario a lo encontrado por Fonseca [21], el cual refiere para esta dimensión un puntaje de 12,57 en una escala de 0 a 28, lo que se traduce en una baja participación social de los participantes del estudio. Diferentes estudios [21, 22] han demostrado que un adecuado apoyo social proporcionado por familiares y amigos disminuye la severa tensión causada por la enfermedad y promueven una mejor recuperación física y mental.

Con respecto a la dimensión psicoespiritual se encontró que el tipo de confort que predomino fue la tranquilidad, que es el nivel intermedio de medición de los tipos de confort (alivio, tranquilidad $\mathrm{y}$ trascendencia) en un $67,7 \%$ de los participantes del estudio, demostrando así que las personas encuestadas se hallan en un estado de calma y satisfacción. Lo anterior concuerda con lo planteado por Jaman [23] quien manifiesta en su estudio que vivir con cáncer significa un proceso de cambios a todo nivel y, sobre todo, en la percepción que tienen los pacientes de sí mismos; en donde la enfermedad les proporciona la oportunidad de reconocer, de reflexionar y tomar conciencia de su "ser", que ahora ha cambiado y que debe reestructurarse desde el encuentro consigo mismo y reporta además, que la mayor parte de los participantes manifestaron sentir confianza y paz consigo mismos, alentarse al saber que cuentan con personas que los quieren y encontrarle significado a la vida.

En cuanto a la dimensión ambiental se encuentra que las personas que participaron en el estudio se encuentran en un nivel de confort que va de la tranquilidad en un $48,9 \%$, estos resultados concuerdan con lo reportado por Jaman [23] en el cual se valora la experiencia de 10 mujeres con diagnóstico de cáncer; donde las participantes manifestaron que la relación con el mundo se ve restringida por los ambientes de la propia casa y el centro de salud, produciéndoles un aislamiento social pero al mismo tiempo afirma que además, esta experiencia les está provocando un nuevo estilo de vivir, una nueva mirada de enfrentarse a la vida, obligándoles de esta manera a vivir con más calma y tranquilidad, dándole oportunidad a nuevas prioridades y nuevas formas de disfrutar la vida.

\section{Conclusiones}

El promedio de edad de los participantes fue de 50 años, de género masculino y la religión con los ritos y costumbres, es utilizada como estrategia para afrontar su condición de enfermedad.

En los participantes del estudio predominó la tranquilidad en la dimensión ambiental y psicoespiritual, condición que les permite presentar una actitud positiva ante su condición de salud.

La trascendencia como el mayor estado de comodidad en que se encontraron los pacientes del presente estudio, les permite estar por encima de los problemas o del dolor propio.

Estudios como el presente permiten al profesional de enfermería conocer el grado de comodidad alcanzado por el paciente con el tipo de cuidado que se le brinda, teniendo en cuenta que la comodidad contempla diferentes dimensiones en el ser humano y Kolcaba desde su propuesta teórica las clasificó en cuatro.

\section{Recomendaciones}

Para la disciplina de enfermería el realizar investigaciones a partir de una teoría de enfermería, permitió en la presente in- vestigación establecer las condiciones de confort del paciente con cáncer como resultado del cuidado. Es necesario continuar con esta línea de investigación para que desde la práctica se generen propuestas que permitan el confort del paciente como resultado del cuidado.

\section{Bibliografía}

1. Farfán, M.d.S.P., Una Visión Integral del Ser Humano. Programa de salud familiar. Ciencia y filosofía de la Salud, 2007(Universidad de Barcelona. 2007. (Consultado 6 de octubre 2010).

2. Alonso, L. and I. Escorcia de Vásquez, El ser humano como una totalidad. Revista Científica Salud Uninorte, 2012. 17.

3. Barrera-Ortíz, L., N. Pinto-Afanador, and B. Sánchez Herrera, La enfermedad crónica: Cuidando a los cuidadores familiares de personas con enfermedad crónica. Grupo de Cuidado Facultad de Enfermería Universidad Nacional de Colombia. Bogotá: Editorial Universidad Nacional de Colombia. Facultad de Enfermería. 2010.

4. Organización Mundial de la Salud. Prevención de las Enfermedades crónicas: una inversión vital. (Consultado 13 de Febrero de 2013). p. 15-16

5. Lence, J.J. and R. Camacho, Cáncer y transición demográfica en América Latina y el Caribe. Revista Cubana de Salud Pública, 2006. 32(3):0-0.

6. Organización Panamericana de la Salud. Registros de cáncer: información para la acción en América Latina y el caribe (Consultado 13 Febrero de 2013).

7. Liga Colombiana contra el cáncer. Cifras estimadas de Incidencia y Mortalidad anual por Cáncer en Colombia. 2008. (Consultado 13 Febrero de 2013).

8. Instituto nacional de Cancerología. Cáncer en cifras 2006. (Consultado 13 Febrero de 2013).

9. Herrera, S., et al., Registro poblacional de cáncer Cartagena de Indias, 2003.

10. Raile, M. and A. Marriner, Modelos y teorías en enfermería. 2011, Editorial Elsevier Mosby.

11. Organización Mundial de la Salud. Cancer Pain Relief and palliative care. (Consultado 13 Febrero de 2013).

12. Peterson, S.J. and T.S. Bredow, Middle range theories: application to nursing research. 2009: Lippincott Williams \& Wilkins.

13. Flórez, M.L.F., Comodidad del paciente hospitalizado en un servicio de cirugía cuando se proveen medidas de comodidad que involucran a la familia. 2001.

14. Ferrandis, E.F., La medición de la comodidad en enfermeras y pacientes oncológicos. Revista Reduca (Enfermería, Fisioterapia y Podología), 2009. 1(2):501-515.

15. Ministerio de Salud. Resolución 8430 de 1993, in «Por la cual se establecen las normas científicas, técnicas y administrativas para la investigación en salud», R.d. Colombia, Editor.

16. Kolcaba, K.Y., A taxonomic structure for the concept comfort. Image J Nurs Sch, 1991. 23(4):237-40.

17. Payán, E.C., S. Vinaccia, and J.M. Quiceno, Cognición hacia la enfermedad, bienestar espiritual y calidad de vida en pacientes con cáncer en estado terminal. 2011. 
18. Gaviria, A.M., et al., Calidad de vida relacionada con la salud, afrontamiento del estrés y emociones negativas en pacientes con cáncer en tratamiento quimioterapéutico. Psicología desde el Caribe, 2007(20):50-75.

19. Finck Barboza, C. and M. Forero Forero, Ansiedad y depresión en pacientes con cáncer de seno y su relación con la espiritualidad/religiosidad. Analisis preliminar de datos. Revista de Psicología Universidad de Antioquia, 2011. 3(1).

20. Gómez Candela, C., et al., Autopercepción de los pacientes con cáncer sobre la relación existente entre su estado nutricional, su alimentación y la enfermedad que padecen. Nutrición Hospitalaria, 2008. 23(5):477-486.

21. Fonseca C, M., et al., Evaluación de la calidad de vida en pacientes con cáncer terminal. Revista chilena de cirugía, 2013. 65:321-328.

22. Baider, L., Cáncer y familia: aspectos teóricos y terapéuticos. International Journal of Clinical and Health Psychology, 2003. 3(3):505-520.

23. Mewes, P.J. and M.a.S. Rivera, Vivir con ca'ncer: una experiencia de cambios profundos provocados por la quimioterapia. Aquichan, 2014. 14(1). 